\title{
Aprendizaje, comunicación e innovación en la formación de maestros de lenguas en Colombia
}

Learning, Communication and Innovation in the Training of Language Teachers in Colombia

Aprendizagem, comunicação e inovação na formação de professores de línguas na Colômbia

\section{Claudia Lucía Ordoñez-Ordoñez* iD orcid.org/0000-0002-9638-5157}

\section{Artículo de investigación}

Revista Colombiana de Educación, N.7 75. Segundo semestre de 2018, Bogotá, Colombia

doi: 10.17227/rce.num75-8108

Para citar este artículo: Ordoñez-Ordoñez, C. (2018). Aprendizaje, comunicación e innovación en la formación de maestros de lenguas en Colombia. Revista Colombiana de Educación, 75, 187-214.

\section{(c) (1) $(9$}

Doctora en educación (Ed.D). Profesora asociada Departamento de Lenguas Extranjeras, Universidad Nacional de Colombia. Bogotá, Colombia. Correo electrónico: clordonezo@unal.edu.co. 


\section{Resumen}

El presente artículo es una reflexión basada en la experiencia reciente de la autora en innovación pedagógica e investigación sobre la educación para el bilingüismo en lengua materna y extranjera y sobre la formación de profesores de lenguas en nuestro país. Está motivada por la convicción de que el mejor profesor de lengua extranjera en nuestros contextos es el bilingüe, hablante nativo de español, que ha vivido las necesidades y problemas del aprendizaje de una lengua extranjera en un ambiente mayormente monolingüe. La autora reflexiona acerca de teoría y práctica en la formación de profesores de lenguas para el bilingüismo que busca el país, argumentando a favor de una educación que incluya y conecte experiencias relacionadas con la verdadera forma como aprende la gente y experiencias innovadoras de desarrollo del español materno y de la lengua extranjera en contextos específicos y para la comunicación efectiva. El objetivo primordial es formar maestros que entienden que no hay maneras fijas, conocidas y probadas de enseñar lenguas, y que exploran y experimentan permanentemente prácticas pedagógicas nuevas en sus aulas, con la intención de adaptarse a muy variados grupos de aprendices y contextos de aprendizaje y siempre mejorar como maestros. El artículo está escrito en español porque la reflexión que contiene aplica en la formación profesional de docentes de cualquier lengua, materna o extranjera.

\section{Palabras clave}

formación de profesores de lengua; desarrollo de habilidades comunicativas; desarrollo de habilidades pedagógicas; aprendizaje y desarrollo de lengua; bilingüismo

\section{Keywords}

training of language teachers; development of communication skills; development of pedagogical skills; learning and language development; bilingualism

\begin{abstract}
The present article is a reflection based on the author's recent experience in pedagogical innovation and research on education for bilingualism in mother and foreign tongue and on the training of language teachers in our country. It is motivated by the conviction that the best foreign language teacher in our contexts is the bilingual, native speaker of Spanish, who has lived the needs and problems of learning a foreign language in a largely monolingual environment. The author reflects on theory and practice in the training of language teachers for the bilingualism sought by the country, arguing in favor of an education that includes and connects experiences related to the true way people learn and innovative experiences in the development of Spanish as mother tongue and of the foreign language in specific contexts and for effective communication. The primary objective is to train teachers who understand that there are no fixed, well-known and proven ways to teach languages, and that explore and permanently experience new pedagogical practices in their classrooms, with the intention of adapting to very varied groups of learners and learning contexts. Always improving as teachers. The article is written in Spanish because the reflection it contains applies to the professional training of teachers of any language, either mother or foreign.
\end{abstract}

\section{Resumo}

O presente artigo é uma reflexão baseada na experiência recente da autora em inovação pedagógica e pesquisa sobre educação para o bilinguismo em língua materna e estrangeira e sobre a formação de professores de línguas em nosso país. Esta pesquisa motiva-se na convicção de que o professor de língua estrangeira em nossos contextos é bilíngue, falante nativo de espanhol, que já experienciou as necessidades e problemas da aprendizagem de uma língua estrangeira em um ambiente principalmente monolíngue. A autora reflete acerca da teoria e a prática na formulação de professores de línguas para o bilinguismo que o país procura, com argumentos a favor de uma educação que inclua e vincule experiências relacionadas com a forma real de aprendizagem de uma língua e experiências inovadoras de desenvolvimento do espanhol materno e a língua estrangeira em contextos específicos e para a comunicação efetiva. O objetivo primário é formar professores que possam entender que não existe um único caminho, conhecido e comprovado para o ensino de línguas, assim como explorar e experimentar permanente práticas pedagógicas em suas salas de aula, visando se adaptar a diversos grupos de aprendizes e contextos de aprendizagem para melhorar sempre como professores. 0 artigo foi escrito em espanhol porque a reflexão apresentada é válida na formação profissional de docentes de qualquer língua, materna ou estrangeira.

\section{Palavras-chave}

formação de professores de língua; desenvolvimento de habilidades comunicativas; desenvolvimento de habilidades pedagógicas; aprendizagem e desenvolvimento da língua; bilingüismo 


\section{Introducción}

La enseñanza de inglés ha sido tema de primera línea en la educación colombiana desde hace ya bastantes años y por razones claras de su importancia como instrumento internacional de comunicación, hasta el punto de convertirse en el centro de interés de un programa del Ministerio de Educación Nacional (MEN) tendiente a promover el bilingüismo español-inglés en todo el país, ahora denominado Colombia Bilingüe (Gómez Sará, 2017). El programa no ha rendido los frutos esperados en lo que respecta a los niveles de inglés de profesores y estudiantes (Gómez Sará, 2017), y sorprende permanentemente la manera como el MEN toma y cambia decisiones sobre estos niveles esperados y sobre política lingüística en general, sin mayor sustento en el conocimiento existente a partir de la investigación.

La autora ha discutido profusamente, por ejemplo, la adopción para la educación pública de acciones que considera erróneas en la educación bilingüe privada, como la introducción de la lengua extranjera en el currículo en una etapa excesivamente temprana a expensas del desarrollo del español, porque no toma en cuenta el conocimiento existente sobre las formas en las que funciona el bilingüismo en diferentes contextos lingüísticos (v. g. Ordóñez, 2008b). Ni siquiera hay conciencia suficiente de que no se hacen bien las cosas desde lo pedagógico en el proceso de buscar el bilingüismo, cuando esto se ha mostrado en nuestros propios colegios privados (v. g. Ordóñez, 2000; 2004b; 2005). De igual manera, el MEN ignora el conocimiento desarrollado en el país sobre los fenómenos educativos, sociales, socioeconómicos y culturales que producen sus políticas (Gómez Sará, 2017; González, 2010), o sobre el desarrollo mismo de la lengua inglesa. Como consecuencia de esto, y a pesar de lo deseable que es el logro del bilingüismo en nuestro país, no hemos encontrado aún formas pedagógicas efectivas de alcanzarlo. Ahora nos corresponde sustentar que este logro es posible, y que lo pueden hacer realidad maestros formados en Colombia.

Y es que la falta de sustento de las políticas del MEN en el conocimiento existente sobre el desarrollo mismo del inglés se nota en una de las más recientes acciones de Colombia Bilingüe: la traída al país de hablantes nativos de inglés, considerados como los mejores tutores en el aprendizaje de esta lengua (v. g. Centro Virtual de Noticias de la Educación, 2015; “200 extranjeros comienzan a dar clases de inglés en colegios públicos", 2015). Medidas como esta se equiparan con las de organizaciones que ofrecen clases con hablantes nativos del inglés como la panacea para el aprendizaje de esta lengua, dirigidas a un público que no tiene por qué conocer, por ejemplo, todo el trabajo teórico e investigativo sobre el inglés como lengua franca y las identidades de los profesores de inglés como lengua extranjera en el mundo entero (v. g. Braine, 2013; Fichtner y Chapman, 
2011; Kirkpatrick, 2014; Moussu y Llurd, 2008; Seidlhofer, 2004) y aun en Colombia (v. g. González, 2010). Este trabajo tiende -entre otros propósitos- a justificar la enseñanza de diferentes variedades del inglés propias de diversas comunidades anglófonas o del inglés como lengua extranjera o segunda lengua, y a sustentar la eficacia de profesores con estas características en la enseñanza del idioma. El Ministerio de Educación parece olvidar también, con políticas como esta, que existen en el país numerosos programas de formación de maestros colombianos de lenguas extranjeras; o al menos ha llegado a la conclusión de que sus esfuerzos para mejorar la formación de los futuros maestros que toman estos programas son inútiles.

Pero además, pensar hoy en día que, en nuestro contexto, el hablante de una lengua es el mejor profesor de esta, aunque -a menudo- no tenga formación como maestro, quiere decir que no se tiene en cuenta el papel de la lengua materna en la formación del futuro sujeto bilingüe (Ordóñez, 2008b). Peor aún, significa que se ignora la importancia de la reafirmación de la propia cultura del futuro bilingüe, a medida que conoce la cultura (o las culturas) asociada a la lengua extranjera; y desde luego, que se ignora la diversidad de un país en el que los contextos de uso de una y varias lenguas son múltiples y complejos (v. g. Bonilla y Cruz-Arcila, 2013) y el profesor de lengua extranjera debe comprenderlos para que pueda tomar decisiones pedagógicas acertadas.

Porque probablemente el mejor maestro de lengua extranjera, con visión de bilingüismo para el contexto de nuestro país, es un maestro que ha vivido aquí el proceso de convertirse en bilingüe, continuando el desarrollo de su lengua y su cultura maternas a medida que aprende la lengua extranjera y conoce las culturas asociadas a ella. De otra manera, perpetuaremos prácticas parecidas a las de los colegios bilingües de élite que le dan importancia reducida al español y se concentran en el desarrollo de la lengua extranjera y en el conocimiento y la celebración de la cultura extranjera (v. g. De Mejía, Ordóñez y Fonseca, 2006; Lastra, 2009), a pesar de las pocas directrices que el MEN ha producido para ellos con base en la investigación (De Mejía y Fonseca, 2008).

Hay que creer en la formación de maestros colombianos de lenguas extranjeras; pero también hay que creer -como lo hacen la mayoría de los académicos de nuestro país que no están de acuerdo con diferentes aspectos del plan de bilingüismo nacional-que esa formación debe seguir mejorando en los programas universitarios que la trabajan. Necesitamos profesores realmente bilingües, o sea personas que puedan usar el español y el inglés de manera efectiva para diferentes necesidades comunicativas auténticas y que estén preparados para ayudar a nuestros jóvenes a alcanzar ese mismo bilingüismo en nuestros contextos. Por ello, en este artículo se busca argumentar la tesis de que debemos asegurar, dentro de esa formación, que los futuros maestros vivan experiencias relacionadas con la forma como 
aprende la gente, la forma como se aprende la lengua en particular y formas de innovar en la enseñanza-aprendizaje de lenguas materna y extranjeras para adaptarse a las necesidades de diversos grupos de estudiantes colombianos, según sus contextos. En el proceso de estos logros, nuestros futuros maestros deben convertirse en verdaderos bilingües. Para lograr esta argumentación se recoge experiencia personal reciente en innovación pedagógica e investigación sobre la educación para el bilingüismo en lengua materna y extranjera y sobre la formación de profesores de lenguas.

\section{Innovación en enseñanza de lengua materna y extranjera}

La experiencia transformadora sobre la que se basa esta reflexión no es tan reciente. Se remonta al comienzo de la década de los 2000, a la dirección de 30 prácticas y tesis de profesores en ejercicio en un programa de maestría en educación creado por la autora en una universidad privada en Bogotá. El propósito del programa era ayudar a los profesionales que lo cursaban a cambiar sus formas de pensar y actuar como maestros de sus disciplinas académicas; para esto debían hacer práctica de la enseñanza en sus salones de clase, algo no convencional en programas de maestría. La razón de la necesidad de esta práctica era que debían crear una innovación pedagógica para sus salones de clase, con base en su reflexión comparativa entre los principios constructivistas del aprendizaje y sus implicaciones pedagógicas — que estudiaban a fondo en el programa—, su experiencia anterior como maestros y su propio aprendizaje durante la vida.

Diseñar y aplicar formas de ayudar a un grupo de estudiantes a aprender de maneras distintas a las que acostumbramos pensar debido a las experiencias que hemos vivido es lo que se considera aquí como innovación o cambio pedagógico; es decir que la innovación es relativa a lo que cada maestro ha hecho hasta el momento como aprendiz. Entre los estudiantes de la maestría en educación en la que trabajamos, varios profesores de inglés como lengua extranjera hicieron su práctica e investigaron el impacto de sus innovaciones en el aprendizaje de sus estudiantes, como tesis para optar al título del programa (González, 2008; Llano, 2009; Moya, 2008); también se trabajó en la dirección de tesis sobre innovaciones en la enseñanza del español (Torres, 2006), incluso en colegios bilingües donde los profesores de español sentían que no había suficiente trabajo académico en la lengua nativa (Jiménez, 2006; Varela, 2008).

Al considerar los resultados formativos que se observaron a nivel de posgrado al ayudar a maestros de lengua en ejercicio a cambiar sus formas de entender el aprendizaje de sus alumnos, a crear nuevas formas de orientar a un grupo hacia el aprendizaje y a verificar los efectos que esto 
puede producir, resultó claro que era innecesario esperar hasta el posgrado para lograr algunos de estos beneficios en la formación profesional. Es así como al pasar a una universidad pública a finales de la misma década, a trabajar con estudiantes de pregrado que serían futuros profesores de inglés, desarrollamos una forma de ayudarles a mejorar sus niveles de uso del inglés hasta que puedan considerarse verdaderos bilingües, y a explorar en su práctica de la enseñanza cómo ayudar a sus estudiantes a aprender esta lengua de maneras distintas a las que ellos mismos vivieron; también hemos logrado aplicar esta misma forma de aprender a enseñar lengua a la enseñanza de habilidades comunicativas académicas en español, a nivel universitario. A continuación se explica de qué se trata, desde la teoría que le da origen hasta la experiencia de su uso y los resultados de la investigación que se ha realizado sobre su impacto en el aprendizaje de estudiantes de inglés y de futuros profesores de la lengua.

Llevamos años abogando por el desarrollo de habilidades lingüísticas, comunicativas, académicas y culturales en español y en inglés, por medio de lo que se define como desempeños comunicativos auténticos (Ordóñez, 2010a). Estas acciones de los estudiantes son diversas y cambiantes, porque se relacionan con sus intereses y sus contextos de aprendizaje. Se han utilizado como actividades simuladas y/o relacionadas con el juego y la imaginación al trabajar con niños pequeños (Guevara y Ordóñez, 2012; Ordóñez, 2008a; 2012; 2016), en actividades de desarrollo auténtico de habilidades académicas tanto en inglés como lengua extranjera, como en español nativo en el colegio o la universidad (Ordóñez, 2008a; 2010b; 2011; Sierra 2017), en comunicación real virtual con pares que usan otras lenguas y pertenecen a otras culturas (Tolosa, Ordóñez y Alfonso, 2015; Tolosa, Ordóñez y Guevara, 2017) y con uso de materiales auténticos de internet, que en esta época de avances tecnológicos es la fuente real más importante y económica de material para aprender a comunicarse en inglés y otras lenguas, tanto para maestros como para estudiantes (Ordóñez, 2015a; 2015b).

Los desempeños comunicativos auténticos son formas de aprender que no tienen nada que ver con los tipos de ejercicios que conocemos los profesores de lenguas desde hace décadas y que son tan comunes en los materiales comerciales de enseñanza; tampoco tienen que ver con la concentración en reglas gramaticales o vocabulario específico. Ni siquiera, con los ejercicios clasificados como los más comunicativos ni con los communicative tasks (Nunan, 1989) que conocemos. La concepción de los desempeños comunicativos auténticos viene de conectar los principios del aprendizaje humano desarrollados por el constructivismo con los del análisis comunicativo de la lengua, tal como se concibieron en sus orígenes. 
A propósito del constructivismo, en un viejo prólogo escrito para un número especial de la Revista de Estudios Sociales de la Universidad de los Andes decíamos que la pedagogía actual había avanzado a partir de la comprensión de la forma como procede el aprendizaje humano, básicamente descrita por el constructivismo. Se mencionaba allí que existía ya mucha evidencia sobre esto en la investigación en educación, pero también se indicaba que la mejor es la reflexión sobre nuestros propios aprendizajes efectivos a través de la vida (Ordóñez, 2004a, p.8).

Parecería que ya en 2004 nos habíamos "quedado" en el constructivismo, y que la cita actual de 2004 es una mayor falta de actualización. Pero la verdad es que no se ha desarrollado teoría más acertada para entender el aprendizaje humano que esta. El constructivismo nos ayuda a visualizar las formas en que aprendemos en la vida real: con base en nuestras experiencias y en las maneras de procesarlas. Estas experiencias pueden incluir el ver y oír a otros haciendo y hablando, y recibir explicaciones, pero sabemos que solo aprendemos cuando hacemos algo real, físico y/o social con lo que nos interesa verdaderamente, e incluso cuando fallamos o cometemos grandes errores con ello. Igualmente, el constructivismo nos permite entender que todo aprendizaje efectivo es producto de procesos normalmente largos de trabajo y reflexión y se traduce en capacidades para hacer cosas con autonomía y participar e influir en las acciones de otros. Esto nos permite igualmente entender que nunca terminamos de aprender nada; que nos mantenernos trabajando en mejorar nuestros conocimientos y habilidades en nuevas circunstancias reales. Todo esto es verificar que los principios del constructivismo se cumplen en el aprendizaje humano y concebir el aprendizaje de una manera menos artificial que la que nos ha presentado siempre la educación.

En aquel viejo prólogo se recordaba que el conjunto de concepciones sobre el aprendizaje que produce el constructivismo proviene de las teorías sobre el desarrollo cognoscitivo generadas por Piaget (1970) y Vygotsky (1978), que definitivamente se conectan. Estas teorías no hablan de aprendizaje en las aulas, sino del que ocurre permanentemente en las personas de manera individual y en sus medios de socialización (Ordóñez, 2004a), y que debe hacernos repensar lo que ocurre en las aulas. Y la comprensión del aprendizaje humano se completa con las ideas de Dewey (1991) sobre la reflexión, que explican la forma como el individuo consciente aprende de lo que hace y le ocurre (Piaget, 1970), y la manera como internaliza (Vygotsky, 1978) ese aprendizaje y lo hace suyo y diferente al de otros.

Para desarrollar esta idea es posible identificar cinco principios del aprendizaje, que ahora se completan con un sexto nada nuevo, todos desarrollados por la investigación psicológica y educativa, durante varias décadas. Estos principios dan soporte a la mayoría de las estrategias 
pedagógicas activas y críticas que caracterizan la práctica pedagógica desde hace ya varios años y que pueden mostrarnos caminos concretos de cambio pedagógico en cualquier área de aprendizaje:

»El aprendizaje es un proceso individual de construcción de significado (Piaget, 1970), que se estimula con la asistencia de otros (Vygotsky, 1978), no es lineal (Rogoff, 1996) y se traduce en una creciente habilidad de comprensión equivalente a la de acción con lo que se sabe (Perkins, 1998).

» Ocurre de manera diferente en cada individuo porque es significativo; es decir, todo lo nuevo se conecta con experiencias y conocimientos desarrollados previamente, adecuados o ingenuos, lo que genera errores de enorme importancia en el proceso de comprender (Ausubel, 1968; Carretero, 2001; Coll, Martín, Mauri, Miras, Onrubia, Solé y Zabala, 1993; Gardner, 1991; Savery y Duffy, 1996).

» Ocurre a partir de la acción (Piaget, 1970) o del desempeño, de modo que se demuestra y avanza por medio de acciones/desempeños que activen y hagan avanzar la verdadera comprensión (Perkins, 1998).

» Se hace más significativo, más dirigido a la comprensión de lo real, cuando ocurre por medio de desempeños auténticos, relacionados con lo que verdaderamente hacen quienes usan el conocimiento en el mundo (Boix-Mansilla y Gardner, 1998; Dewey, 1945; Hetland, Hammerness, Unger y Wilson, 1998; Ordóñez, 2010a; Perrone, 1998).

» Se estimula y ocurre naturalmente al poner las comprensiones individuales en interacción inteligente con las de otros (Bruffee, 1999; Dickelman y Greenberg, 2000; Savery y Duffy, 1996; Vygotsky, 1978).

» Se desarrolla, conecta y consolida en la reflexión individual sobre la experiencia y el aprendizaje propios y de otros (Dewey, 1991).

Dos años después de este viejo prólogo, se publicaba su continuación, a manera de artículo teórico general sobre pedagogía en la Revista Ciencias de la Salud de la Universidad del Rosario, indicando que aunque algunos de estos principios son conocidos en la educación desde hace casi un siglo y los más recientes desde hace más de una década en los trabajos del Proyecto Zero de la Escuela de Educación de Harvard, no se han logrado llevar a la práctica de manera sistemática y masiva, por lo menos en nuestra educación colombiana. Y es que si lo hiciéramos, muchas cosas que ocurren actualmente no ocurrirían o lo harían de manera muy diferente (Ordóñez, 2006). Esto es cierto para la enseñanza-aprendizaje de todas las áreas académicas, pero especialmente para las de lenguaje, porque la adquisición del lenguaje es un ejemplo perfecto de aprendizaje como 
proceso, que ocurre como construcción individual y social a partir de un desempeño auténtico característico y muy complejo: la comunicación (Ninio y Snow, 1996).

Desde que Hymes desarrolló el concepto de competencia comunicativa (Hymes, 1971, en Brumfit y Johnson, 1991), sabemos que entender la naturaleza del lenguaje más que como sistema de partes y reglas es mucho más productivo para comprender su adquisición y su aprendizaje (Ordóñez, Barriga-Villanueva, Snow, Uccelli, Shiro y Schnell, 2001). El lenguaje es un instrumento de expresión y negociación de significado; y el significado y los propósitos de la comunicación se originan en el contexto sociocultural en el que se produce, entre participantes en esa comunicación. Estos conceptos comunicativos fueron aplicados específicamente al aprendizaje del inglés como lengua extranjera desde muy al inicio de la conformación de la teoría (Brumfit y Johnson, 1991; Widdowson, 1978), pero no parecen haber logrado hacer mayor mella, porque al analizar libros de texto de enseñanza de inglés, las formas de enseñar siguen basándose en secuencias de estructuras gramaticales y vocabulario, para llegar al final a la comunicación.

Por su parte la investigación en adquisición de la lengua materna indica hace mucho tiempo que ella ocurre por la orientación humana y la necesidad de comunicación auténtica con otros, guiada igualmente por el contexto sociocultural en el que ocurre, por la naturaleza de los participantes en la comunicación y de sus relaciones, y por los propósitos comunicativos que persiguen (v. g. Ninio y Snow, 1996; Ordóñez et al., 2001). No hay razones para pensar que esto sea diferente en la lengua extranjera, excepto porque al iniciar su aprendizaje se tiene ya amplio conocimiento de una propia, el cual debe ayudar en la adquisición de la extranjera por razón de los mismos principios constructivistas que describen el aprendizaje humano (Ordóñez, 2008b).

En efecto, si la educación formal en lenguas verdaderamente estimulara el desarrollo de habilidades de comunicación, y según nuestras políticas educativas el bilingüismo, trabajaría en la ampliación de los repertorios lingüísticos de los aprendices en su lengua nativa, en este caso el español, y en la extranjera (Ordóñez, 2008b), por medio de desempeños auténticos relacionados con la comunicación que realizan las personas comunes en la actividad diaria y a través de los medios, y también con la que se realiza en contextos específicos de experticia y especialización, como los entornos académicos y los literarios, según sus intereses y necesidades; y lo haría en ambas lenguas desde los niveles principiantes. En contextos académicos el lenguaje permite la comprensión y el desarrollo del conocimiento en todas las ciencias básicas, humanas y sociales, la conexión entre conocimientos de diferentes áreas y la comunicación de conocimiento a las comunidades especializadas y no especializadas. En la literatura el lenguaje permite la 
acción de la imaginación en la creación y comprensión de personajes e historias y la comunicación de sentimientos, emociones, valores y toda la riqueza y diversidad humanas (Ordóñez, 2008a).

Los desempeños auténticos nos relacionarían con materiales lingüísticos igualmente auténticos para las clases de lengua materna y extranjera. Para las clases de lengua extranjera, estos materiales se han venido usando desde hace ya bastante tiempo (Insuasty, 2008), junto con actividades comunicativas auténticas entre personas y grupos. Estas actividades permiten una interacción eficaz en contextos específicos, tanto en situaciones presenciales como virtuales (Ordóñez, 2008a). A nivel más específico se pensarían desde la descripción compleja que ha hecho el enfoque comunicativo de las habilidades lingüísticas desde hace treinta años (Widdowson, 1978).

La descripción de Widdowson (1978) nos permite entender que las habilidades comunicativas no son simplemente cuatro. Tanto oralmente como por escrito hay producción e interpretación a diferentes niveles de significado y de forma individual, unidireccional o recíproca (Widdowson, 1978). Así podemos pensar, por ejemplo, en ocasiones comunicativas orales en las que priman el escuchar con intención de comprender (al oír radio o una presentación académica), el hablar con una audiencia específica en mente (discursos y presentaciones públicos para propósitos diversos o comunicación por medio de la radio o la televisión) o la conversación (hablar y comprender en forma recíproca). En el medio escrito, por su parte, pueden identificarse ocasiones comunicativas en donde debe ocurrir la lectura individual con intención de comprender, o con respuesta escrita para el autor o para otros (común en el medio académico o en algunos tipos de publicaciones); la escritura individual con propósitos de expresión personal hecha pública (en la expresión literaria), con lectores específicos en mente (también característica de las publicaciones escritas o virtuales) y la correspondencia o escritura recíproca (común hoy en internet).

Si la educación en lenguas verdaderamente permitiera y estimulara el desarrollo de habilidades de comunicación, los currículos darían el tiempo necesario para que sucediera ese desarrollo: todo desempeño comunicativo que se trabajara debería aparecer de nuevo, repetidamente, conectarse cada vez con más desempeños ya trabajados y usarse en situaciones más variadas. Los currículos deberían indicar conexiones entre todos los desempeños comunicativos practicados y ninguno debería terminar de ser aprendido. $Y$, desde luego, los desempeños auténticos tendrían que desarrollarse en el nativo español, desde antes de que comience el desarrollo de la lengua extranjera; y cuando esto ocurra, los desarrollos deberían ser paralelos (Guevara y Ordóñez, 2012; Ordóñez 2006, 2008a, 2010b, 2011, 2012, 2016). 
Si la expresión "construcción de significado" tuviera verdadera importancia en la educación en lengua, los maestros deberíamos estar concentrados en la actividad significativa de quien aprende, no en lo que debemos hacer nosotros para enseñar trozos constitutivos de lengua, cómo y cuándo utilizaremos qué partes de los libros de texto con los estudiantes y qué partes deberán hacer de "tarea". Deberíamos pensar más bien en cómo sería bueno poner a los estudiantes a comunicarse verdaderamente desde el comienzo de su aprendizaje, para ir acercándose cada vez más a la comprensión y la producción adecuada del lenguaje en su uso en la realidad. Deberíamos pensar constantemente en experiencias durante las cuales pudiera "verse" ese proceso de construcción de significado en los aprendices, cosa fácil de hacer cuando trabajamos con desempeños bien determinados y posibles de describir, como leer un tipo específico de escrito buscando el propósito del autor y la manera como organiza y conecta la información (¿qué información?) para lograrlo; o escribir un texto con el mismo tipo de propósito en forma efectiva. El problema es que la mayoría de lo que queremos que nuestros alumnos aprendan en lengua, lo que está en los currículos, no es esto sino "teoría" sobre la lengua y cómo funciona, información, contenido. Esto siempre es primero, antes de que ellos puedan utilizar esa información en la práctica, para comunicarse.

Si nos preocupáramos menos por juzgar las fallas de nuestros estudiantes y más por entender en qué consisten sus habilidades y conocimientos actuales -incluso en la lengua materna aunque nos propongamos enseñar una extranjera-, podríamos usarlos como arranque hacia la comprensión de lo nuevo, y probablemente lograríamos más en términos de aprendizaje. Para dar mayor soporte a los aprendices en sus procesos de comprensión, los desempeños de comunicación auténtica deberían permitir que estos conocimientos previos, en cualquier lengua, salieran a flote y fueran conocidos por todos los involucrados en el aprendizaje (maestro, compañeros y los mismos aprendices), de modo que se usaran, discutieran y revisaran. Deberían emplearse para aprender lo nuevo, porque lo nuevo no se entenderá apropiadamente hasta que sus conexiones con el conocimiento previo se manejen y se revisen, corrijan y completen (v. g. Gardner, 1991).

La convicción de este "deber ser" nos lleva a definir algunos principios comunicativos de interés, al pensar en la creación de innovaciones pedagógicas en la enseñanza de lengua extranjera:

» Todo aprendiz puede adquirir habilidades de uso de una lengua extranjera siempre y cuando tenga auténtica necesidad comunicativa de hacerlo.

» Resulta importante que se hayan desarrollado ya habilidades efectivas de comunicación en la primera lengua porque ellas sirven de base efectiva para su desarrollo en la extranjera. Si no están 
eficazmente desarrolladas, y aun si lo están, la conexión de lo que se aprende en la lengua extranjera con lo que ya se sabe o se está desarrollando en la primera constituye una clave de éxito en la lengua extranjera y puede ser igualmente un impulso importante en el desarrollo o la mejora de las habilidades comunicativas en la lengua materna.

» La lengua se aprende al usarla para comunicarse en cualquier medio, oral, escrito o electrónico, en la vida diaria y en las ocupaciones humanas de esparcimiento, expresión, estudio, trabajo o ciencia.

» Al participar en la comunicación se produce e interpreta discurso, que se caracteriza por presentar ideas conectadas y organizadas a partir de propósitos comunicativos, desarrollados por medio de oraciones estructuradas y palabras asociadas (Widdowson, 1978) y, agregaríamos, palabras y estrategias asociadoras.

» Las características de un discurso particular dependen del contexto sociocultural en que se produce, que determina las características y relaciones entre los participantes en la comunicación y sus propósitos. Esto determina el lenguaje utilizado y sus características estructurales y formales, porque quien lo produce escoge los elementos lingüísticos que considera efectivos para lograr lo que se propone. Entender los propósitos de los participantes en la comunicación es clave para entender no solo los mensajes, sino la forma como se usa el lenguaje para expresarlos.

» La parte formal del lenguaje, pues, corresponde al discurso y sus características, que a su vez corresponden a quienes los producen e interpretan y sus relaciones, de modo que debe trabajarse a medida que quien aprende a comunicarse, se comunica.

\section{Una innovación y una experiencia definitivamente posibles}

Es posible encontrar alguna evidencia de que se puede formar aprendices de lengua y aprendices de docentes para el bilingüismo con una innovación metodológica adaptable a diferentes grupos de estudiantes, que sigue estos principios tanto del aprendizaje como de la comunicación; se ha hecho al crear e investigar el impacto de una metodología de enseñanza-aprendizaje que maneja materiales auténticos producidos para la comunicación (Ordóñez, 2015a). Al usar esta metodología en cursos reales ofrecidos para diversos grupos de aprendices se han dirigido también prácticas de enseñanza de futuros profesores, quienes han actuado como tutores que facilitan la comunicación y ayudan a los estudiantes a comunicarse 
auténticamente, a la vez que les ayudan a entender cómo funciona la lengua para poder hacerlo. También se ha investigado el impacto de esta experiencia en los practicantes (Ordóñez, 2015b).

La creación de la metodología obedeció originalmente al reto de proporcionarles a estudiantes de la carrera de Lenguas Extranjeras experiencias auténticas de comunicación con la lengua inglesa desde el primer momento de su aprendizaje de este idioma. Todos nosotros somos audiencia para producciones televisivas internacionales que se transmiten directamente a nuestras casas por los canales de televisión por cable y aun por los nacionales, y gracias a internet podemos enfrentarnos a materiales auténticos producidos en inglés con gran facilidad. Los jóvenes, con mayor razón lo hacen. Igual ocurre con las estaciones de radio internacionales que permiten acceso amplio a sus programas digitales. El propósito de la metodología era doble: buscar que los estudiantes avanzaran rápidamente y con eficacia en el conocimiento y uso de la lengua extranjera enfrentándose a estos materiales y, a la vez, lograr que tuvieran un referente experiencial para entender que es posible aprender y enseñar lengua extranjera de manera novedosa y desde la verdadera comunicación.

Se reunió para este efecto a todos los estudiantes que entraban a la sección de inglés de una universidad pública en Bogotá, alrededor de 60, para luego dividirlos en grupos pequeños de niveles parecidos de inglés; este pequeño tamaño de grupo (un máximo de 7 integrantes) podía garantizar que todos participaran realmente en su aprendizaje; pero además aseguraba que podía reunirse en un solo curso un número suficiente de tutores-practicantes en entrenamiento que atendieran individualmente "estaciones" definidas por diferentes tipos de desempeños auténticos comunicativos y materiales auténticos de internet (videos, podcasts, textos visuales y escritos, entre otros). Estos materiales debían ser escogidos especialmente para este grupo de estudiantes, que iniciaba su estudio general del inglés para llegar a ser docentes de la lengua.

Cada "estación" debía exigir a los participantes esfuerzos auténticos de comprensión oral, lectura y expresión oral y escrita en la lengua extranjera, con guía y soporte mínimos por parte de un tutor. Los tutores debían manejar formas reales de aprender y de ayudar a otros a aprender, que no habían estudiado, observado o manejado antes en su formación como maestros en la carrera. Básicamente debían analizar cada material con los principios de análisis del discurso que desarrollaron quienes describieron la lengua como discurso en sus inicios y aplicaron esta descripción a la enseñanza del inglés como lengua extranjera (Castilla y Lobo-Guerrero, 1979; Widdowson, 1978), y convertir ese análisis en decisiones pedagógicas que les permitieran apoyar a los estudiantes en su aprendizaje. 
Las metas que debía buscar este apoyo son comunicativas: en comprensión oral y escrita, los estudiantes debían convertirse paulatinamente en audiencia no solo capaz de usar la lengua extranjera para relacionarse directamente con los materiales, sino capaz de extraer de ellos y usar para diferentes propósitos cada vez más significado; en producción oral y escrita, debían convertir ese aprendizaje proveniente de la comprensión en formas de lograr múltiples propósitos comunicativos en contextos y para propósitos diversos de escritura y comunicación oral. Y en todas las instancias comunicativas, la guía de los tutores debía llevar a los estudiantes a entender la forma como funciona la lengua para producir significados y para lograr propósitos comunicativos.

Se organizaron "estaciones" específicas para este grupo de estudiantes inicial (televisión, radio, lectura, escritura, música y karaoke, cocina, cómo hacer cosas, juegos de rol comunicativos, cuentos e historias, foro virtual, presentaciones orales, entrevistas, comité editorial, viajes y culturas, rimas y juegos de lengua, etc.), pero la metodología exigía modificarlas o crear otras y escoger materiales totalmente diferentes para cada una, de acuerdo con las necesidades y propósitos del grupo de estudiantes al que se dirigía el curso. En efecto, desde su creación en el primer semestre de 2012 y hasta el primero de 2014, el curso se dirigió a estudiantes principiantes que serían finalmente profesores de lenguas; pero desde entonces el curso se ha plasmado en diferentes versiones para muy diferentes grupos de estudiantes.

Entre agosto de 2013 y diciembre de 2014, por ejemplo, la metodología se utilizó en un instituto de enseñanza de inglés creado por un grupo de socios que nos incluyó, con algunos de los graduados jóvenes que habían participado en la creación del curso, tutores formados en esta perspectiva y para público en general con diferentes razones y necesidades en el aprendizaje del inglés. Allí desarrollamos un sistema para que los estudiantes pasaran por seis niveles de dificultad en el uso de la lengua en su proceso de aprender a comunicarse con materiales auténticos; igualmente desarrollamos un programa especial para niños de ocho a catorce años y algunos niveles de material y enseñanza de español para extranjeros, que se puso a funcionar con un grupo de mujeres adultas estadounidenses.

En el mismo diciembre de 2014 comenzamos, con algunos de los tutores más avezados, a entrenar a treinta maestros de los colegios en concesión de Alianza Educativa ${ }^{1}$ para implementar esta metodología en todas sus secciones de bachillerato a partir de enero de 2015, aunque no en las condiciones ideales en las que fue creada y funcionó tanto en la Universidad Nacional como en el instituto de lenguas. En efecto, solo fue posible trabajar con grupos de catorce o quince estudiantes, demasiado grandes para la concepción de la metodología, con una pobre intensidad 
semanal de cuatro periodos de menos de una hora y con maestros que no contaban con una buena formación pedagógica ni con un nivel de inglés adecuado. Sin embargo, desarrollamos currículo para niños y adolescentes jóvenes y para dos años de formación principiante en inglés, que replicamos en todo el bachillerato de los cinco colegios. El proceso se sostuvo durante el año académico 2015, y los estudiantes comenzaban a acomodarse a la metodología y a demostrar aprendizaje, cuando la Alianza quiso tomar el control de la evaluación de los estudiantes y maestros, cosa que rechazamos. El proceso quedó trunco, aunque comenzaba a mostrar un interesante nivel de éxito también en el desarrollo profesional de maestros en ejercicio.

La metodología volvió al nivel universitario durante el primer semestre de 2016, esta vez para un grupo piloto de 60 estudiantes avanzados de diferentes carreras ofrecidas por la universidad donde fue concebida, que cursaban los niveles 3 y 4 intensivos de inglés. Estos son los dos últimos posibles en el programa general de lengua extranjera de la institución, y la reunión de los dos se hizo para permitir una intensidad de trabajo de 2 horas por día, 4 días de la semana. El éxito del piloto entre los estudiantes hizo que el programa solicitara que nos encargáramos totalmente de esos dos niveles en su modalidad intensiva, ofreciendo 6 horarios diarios del curso, desde las 7 a. m. hasta las 8 p. m. El ejército de tutores necesario para esta labor se logró con un tutor-coordinador experimentado para cada horario, entre 5 y 8 docentes practicantes de la carrera de Lenguas Extranjeras y un número restante de estudiantes auxiliares con buen nivel de inglés que entrenamos como tutores principiantes, menos intensivamente que a los practicantes. Estos últimos contaban con trabajo semanal de búsqueda y análisis de material bajo nuestra dirección, observación y evaluación/conversación permanentes sobre él; y todos contábamos dentro de nuestras horas de trabajo con tiempo de observación/conversación con practicantes y tutores principiantes.

Siguiendo la tendencia actual nacional, la Universidad Nacional abolió todas las versiones de estos cursos a partir del segundo semestre de 2017, para reemplazarlos por cursos tradicionales de 30 estudiantes con asistencia de ayudantes extranjeros traídos con becas y fondos especiales; con esto ha logrado aumentar sus estadísticas de internacionalización, necesarias para la acreditación institucional internacional. En la actualidad dictamos solo una nueva versión de inglés para propósitos específicos (ESP, por sus siglas en inglés) dirigido a estudiantes de la Facultad de Ingeniería. En esta versión, financiada independientemente por la facultad, cuatro tutores-coordinadores han podido seguir desarrollando la metodología con nuestra asistencia, primero con un grupo de estudiantes de posgrado y 
profesores de la facultad y luego con estudiantes de los mejores promedios en la carrera, que tienen perspectivas de movilidad internacional para la continuación de sus estudios.

En su periodo inicial 2012-2014, y entre sus varias versiones con cuatro grupos diferentes de estudiantes de lenguas extranjeras, se llevó a cabo investigación cualitativa y mixta sobre el impacto del curso en el aprendizaje de lengua de los estudiantes de inglés y en el aprendizaje pedagógico de los profesores practicantes (Ordóñez, 2015a; 2015b). La investigación cuantitativa sobre el aprendizaje de los estudiantes de inglés mostró avances significativos en las habilidades productivas oral y escrita. Estos resultados estadísticos, sin embargo, no están basados en tests estandarizados sino en evaluaciones desarrolladas para el curso por el grupo de coordinadores y practicantes, y revisadas y editadas por su profesora, lo cual se indica como limitación del estudio, al igual que el hecho de que solamente se analiza el desarrollo de dos habilidades comunicativas amplias. A pesar de esto, la triangulación con el análisis cualitativo corroboró en mucho estos aprendizajes al mostrar conciencia de dichos avances en la mayoría de los estudiantes; además, se reveló un resultado positivo muy deseado dentro de los objetivos del curso, consistente en que algunos estudiantes hablaron del desarrollo de la conciencia de sus propias posibilidades de mejorar su inglés por medio de actividades autónomas (Ordóñez, 2015a).

Por su parte, la investigación cualitativa sobre el aprendizaje de dos tutores coordinadores recientemente graduados y cinco tutores practicantes reveló, en todos, grandes cambios en sus concepciones de lengua y de lo que se enseña al enseñar lengua, de su rol y el de sus estudiantes en términos de las relaciones que deben establecerse entre ellos y las acciones de cada cual, y de lo que es un currículo en una clase de lengua. Igualmente todos describieron técnicas específicas que desarrollaron para lograr el uso de verdaderas habilidades de comunicación en los estudiantes al leer, hablar y escribir y para evaluar su aprendizaje. Finalmente dieron cuenta de grandes avances en sus propias habilidades comunicativas en inglés y del desarrollo de una visión global del aprendizaje de lengua y de lo pedagógico, que vivieron y calificaron como transformador. Atribuyeron además su conciencia profunda de su propio aprendizaje a un proceso continuo que partió siempre de la reflexión, o sea un aprendizaje totalmente consistente con la descripción que hace de él el constructivismo (Ordóñez, 2015b).

Más allá de estos dos estudios, los cuales la autora dirigió y en los cuales participó solo como profesora de práctica de la enseñanza, la evaluación positiva de impacto se basa en la observación de clases por parte de ella y de los tutores, en los comentarios informales de estudiantes y maestros y en informes de evaluación de cursos producidos por el 
programa de Lenguas de la universidad pública en la que se creó y se ha usado la metodología. En el segundo semestre de 2017, una de nuestras estudiantes de la Maestría en Educación emprendió una nueva investigación cualitativa -actualmente en progreso- en la que se interesa más que todo por caracterizar el curso y distinguirlo en todos sus detalles de las prácticas tradicionales de enseñanza del inglés. Es un estudio puramente cualitativo, basado en entrevistas semiestructuradas, reforzadas por una segunda entrevista de profundización a los seis tutores más avezados en esta metodología, que han recibido entrenamiento desde el comienzo mismo de su uso, han participado como tutores del curso en varias de sus versiones y han sido además coordinadores y formadores de otros tutores en el proceso que hemos seguido desde 2012. Ellos autorizaron utilizar los datos de las primeras entrevistas para este artículo, lo que permite refrescar las descripciones del impacto que causa el curso en quienes tienen la experiencia de dictarlo y, a través de sus ojos, en los estudiantes de inglés. A continuación se presenta en la forma de una narración continuada en boca, principalmente, de los tutores.

En primer lugar, los tutores describen su experiencia al llegar al curso y lo presentan de manera general, desde su punto de vista:

He trabajado muy poco con otros métodos, la verdad, y no me sentía cómodo [con ellos] porque no sentía que los estudiantes estuvieran aprendiendo tanto como [correspondía al] esfuerzo que yo pudiera hacer... Podrían aprender mucho más de otra manera; el problema era que yo no sabía cuál. Yo había trabajado con clases particulares, [...] así como con "bueno, vamos a revisar qué es el presente simple",... un método completamente enfocado en la gramática... Supuestamente después del presente simple se tiene que enseñar el continuo, y después el perfecto, y después y al mismo tiempo está el pasado simple, y así... Yo la verdad me sentía muy frustrado tratando de enseñar así...

Para cuando yo llegué a mi práctica docente, ya había pasado por las clases de Didáctica y llegué completamente desilusionado y asustado, porque dije: "bueno, yo elegí la docencia como mi carrera y no sé qué estoy haciendo como docente, porque no veo que el trabajo que se haga normalmente entrañe un aprendizaje real de lengua"... Afortunadamente [en la práctica], trabajando con desempeños auténticos, no solo diría que me cambió la perspectiva de aprendizaje de lengua sino [la de] aprendizaje en general, y además la perspectiva de vida, porque ya dije: "bueno, tengo un trabajo que puedo hacer de una manera más coherente y más efectiva", no digo (y ese es el cambio del paradigma) para yo enseñar inglés, sino para ayudar al estudiante a que aprenda inglés.

Cuando hablamos de enseñanza tradicional... y organización de clase, hablamos de lesson plan, un plan que va a ser más como una tabla de qué se va a hacer en cada momento de la clase. Eso era lo que me 
parecía absurdo de eso, esta parte de la clase se tiene que demorar de 5 a 10 minutos, esta parte de la clase de 20 a 25 y hablamos de que al principio les damos... un vocabulario en el tablero en el que vamos a trabajar ese día o les vamos a dar unas estructuras que después vamos a practicar y que después ellos van a producir. [En cambio aquí básicamente no diseñamos] una clase, [sino que hacemos] un análisis profundo de material y [tomamos] una serie de decisiones pedagógicas [y tenemos] preguntas listas para trabajar con los estudiantes... Entonces no vamos a tener ese tipo de secuenciación, porque todo el tiempo vamos a estar analizando, trabajando y ayudándole a los chicos a que analicen el texto...

Primero que todo entendemos la parte de sonido, qué palabras están diciendo; de ahí armamos la idea completa, a partir de nuestras preguntas, y ahí si vemos "bueno, esta es la idea más o menos general; esto es lo que se está queriendo decir en este pedacito del texto, con qué palabras [y de qué forma] lo dice..." (Tutor 1, entrevista en profundidad, noviembre de 2017).

[Este es] un método que a mí me motiva mucho para aprender inglés; lo describiría como un método apasionante que le permite a uno explorar y entender el fenómeno de la comunicación, no solo en inglés, sino entender la comunicación como una actividad humana... Eso es una de las cosa más apasionantes que yo encuentro de este método: que trasciende las fronteras del código y te hace encarar si puedes o no comunicarte, si sabes o no comunicarte, si sabes entender a otra persona o no a través de un texto... Lo describiría no como la única forma para enseñar lenguas, pero sí la más chévere.

Hay muchas formas de enseñar y mucha gente ha escrito cosas sobre enseñar... pero yo por mi experiencia de las clases que tuve y las clases que he dado tradicionales, me quedo con este método... Los desafíos que me impone este método me interesan más y tienen que ver más con mi gusto personal que los desafíos que me impone una clase tradicional: a mí no me interesa ver cómo hago para controlar un grupo de $25 \ldots$ ese es un desafío que sinceramente no está dentro de mis desafíos personales, porque no me interesa ser el centro de atención, porque no me interesa ser la más chistosa... no me interesa desarrollar esas habilidades. Me interesa el desafío de qué decisión tomo para que este grupo de 10 personas se comunique mejor con este material. Ese desafío sí me interesa, porque yo también estoy enfrentando ese desafío, yo también estoy analizando ese material, yo también me estoy equivocando para entender... (Tutor 2, entrevista en profundidad, noviembre de 2017). 


\section{Y luego hablan en detalle del aprendizaje que han visto en sus estudiantes:}

Es un método muy bien pensado, muy bien planeado; he visto los resultados en los estudiantes. La verdad que... no se me ocurre otro método en el que ellos podrían alcanzar tanto avance en tan poco tiempo. Hicimos un tiempo un examen de clasificación... al principio del curso y al final del curso, y el avance en lengua en los estudiantes era impresionante; teníamos estudiantes que subían hasta 2 niveles en el Marco Común Europeo en 4 meses, absolutamente sorprendente... De verdad tendría que ser una cosa completamente diferente, como para decir "ok, se puede de esta manera o de esta otra". Se necesitaría otro genio loco que se invente eso... (Tutor 5, entrevista en profundidad, noviembre de 2017).

El curso [basado en] desempeños auténticos tiene unos resultados muy, muy buenos... [porque...] no solo se aprende lengua, sino que se aprenden estrategias intelectuales... estrategias de lectura, de escritura, de desarrollo del lenguaje, de la expresión escrita; bueno, el performance oral; ... como que eso lo hace muy, muy efectivo y muy exitoso [por el] el trabajo con desempeños auténticos. Mientras que tú trabajas con un libro, es mucho más difícil hacer ese trabajo fuerte de las estrategias porque no son materiales auténticos; porque todo está tan desglosado para el estudiante, que no permite ese desarrollo intelectual y cognitivo que requiere uno en la vida real para leer y para escribir (Tutor 3, entrevista en profundidad, noviembre de 2017).

Otro aprendizaje que yo diría que tiene esto... es... con la percepción, porque en los métodos tradicionales, las 4 habilidades se dividen demasiado; como que se dice que esto es speaking, que esto es listening, esto es reading y esto es writing, y no se hace una conexión completa, que es la realidad de la lengua... La organización por estaciones nos permite trabajar intensivamente... en la habilidad perceptiva, y eso es algo que nos han dicho mucho los estudiantes... en la evaluación docente, como también verbalmente: "es que yo ahora me siento y entiendo cosas de verdad... y no solo palabras sueltas, sino que soy capaz de entender los propósitos". Hablamos de unidades de sentido completo, y eso es lo básico que queremos que los chicos entiendan... También nos hemos dado cuenta de que son capaces de entender la globalidad de un texto en términos de [su] propósito y de cómo cada una de las partes [aporta] a ese propósito... Son capaces de ver la lengua por lo que es: una realidad comunicativa y no un conjunto de estructuras gramaticales. [Entienden] que tiene ambas cosas:... la realidad comunicativa se expresa a partir de estructuras gramaticales, pero... tanto profesores como estudiantes Ilegamos... a la misma conclusión: que [las] estructuras son importantes porque... están ligadas a un uso real en la comunicación, y eso es algo que definitivamente no he podido ver en otros métodos de enseñanza. (Tutor 1, entrevista en profundidad, noviembre de 2017). 
[Les encanta trabajar con material auténtico] porque muy pocas veces han tenido esa oportunidad en sus clases; porque siempre se han enfocado es en la gramática... y memorizar y escribir y llenar el espacio... con el vocabulario; pero cuando uno les pone ya el reto de que escuchen material que no es del libro [donde] está modificado para que uno [diga] "ah, sí ya, tan chévere, entiendo todo",... ellos ven el reto. A veces es desmotivante para ellos, pero ahí es donde entra el trabajo de uno como [tutor]... Y se van acostumbrando, y les gusta... y ellos sienten una evolución... Lo más difícil para ellos es sobre todo entender diferentes acentos; entonces [tratamos] de traer material... de diferentes nacionalidades, de diferentes temas, con diferentes ritmos de habla; entonces les gusta porque es un reto [que] los pone de verdad a escuchar y a ponerle lógica a la cosa... Es como un entrenamiento del oído bien chévere que muy pocas veces han tenido (Tutor 6 , entrevista en profundidad, noviembre de 2017).

Ellos mejoran el nivel de lengua y, además de eso, se podría decir que aprenden cómo enfrentarse a ese tipo de material [auténtico]. Generalmente en una clase de lengua uno tiene al profesor como "salvavidas"; acá aprenden que no:... [en] quizzes y exámenes... ellos tiene que enfrentarse a todo eso solitos... Entonces ellos aprenden ... qué de pronto es clave, qué de pronto no... Para mí eso ya es una herramienta gigante para [poderse] enfrentar a una lengua... en contextos reales (Tutor 5, entrevista en profundidad, noviembre de 2017).

La gente se ve más motivada al final de los cursos; ... suelta mucho el oído porque hacemos un trabajo intensivo en esa parte, que es la más descuidada en otro tipo de métodos... Les enseñamos mucho cómo funciona la lengua en general... Esos son comentarios que hemos recibido, como que "este texto argumentativo que ustedes trataron de ayudarme a escribir en inglés fue una guia para escribir mi ensayo en español de tal clase" ${ }^{\prime \prime}$.. Aprenden mucho de la cultura general... cosas muy interesantes todos los días ... Y sobre todo [aprenden] que no necesitan un profesor o la clase fija de inglés para poder aprender un idioma; como que se pone en evidencia la responsabilidad que tiene cada persona sobre su propio desarrollo lingüístico (Tutor 4, entrevista en profundidad, noviembre de 2017).

Hablan de manera especial de la motivación, en el sentido de que los estudiantes cambian sus concepciones negativas sobre el aprendizaje del inglés y sus propias habilidades para lograrlo.

Me gusta mucho ver cómo ese tiempo que uno le invierte al curso se ve recompensado en la impresión y en el impacto que causa en los estudiantes, y me alegró mucho... ver cómo los estudiantes que tenían mucho miedo del inglés, que lo odiaban porque siempre los regañaban los profesores o porque se burlaban, al final nos agradecían y nos decían que el curso les cambió lo que pensaban del inglés... Para muchos 
aquí es como el "Coco"... y a muchos les hemos ayudado a cambiar esa percepción y les damos más confianza y escriben muy buenos comentarios del curso; entonces... valió la pena todo el esfuerzo, toda la búsqueda de material, todo el análisis, toda la "reunidera", todas las observaciones, porque los estudiantes lo aprecian... Ellos valoran mucho la metodología, lo de las estaciones, lo de los grupos pequeños... incluso dicen que ojalá se pudiera ampliar no solo en los cursos de inglés sino a otros cursos de la universidad ... y decían que ojalá el curso no fuera para los niveles 3 y 4 sino para todos los niveles de lengua... es bonito ver que los estudiantes quedan impactados... y quedan contentos y agradecidos (Tutor 6, entrevista en profundidad, noviembre de 2017).

Yo recibo un millón de evaluaciones de los estudiantes diciendo que aprenden, que su percepción del inglés cambió, que ahora les gusta; si tú analizas los comentarios que hay al final del curso... pues son muy positivos... ¡Cómo no va a valer la pena investigar un curso que tiene una favorabilidad tan grande entre los estudiantes... uno que sí funciona! (Tutor 2, entrevista en profundidad, noviembre de 2017).

Y finalmente, hablan de su propio aprendizaje al enseñar con la metodología de desempeños auténticos:

Uno aprende mucho; entonces no es solo un método para el estudiante sino para nosotros. Aprendemos no solo de nuestras discusiones entre profesores, sino de la preparación del material; todos los días aprendemos alguna palabra, alguna bobada... Yo creo que yo solté mucho el oído... Y me gustó mucho la forma de evaluar que nos enseñó la profe, porque nunca había usado [una] matriz de evaluación; eso fue nuevo para mí. Y teóricamente terminé leyendo mucha cosa... sobre la competencia comunicativa, que es lo que se busca desarrollar con esto, no la gramatical únicamente. Entonces yo creo que el mayor aprendizaje... es... que me abrió mucho las perspectivas de lo que hace o debería hacer un profesor de lengua, que no es solo 2 jay me sé la regla y hablo bonito!" (Tutor 4, entrevista en profundidad, noviembre de 2017).

Yo siento que antes de este método yo no sabía leer de verdad; yo hacía una actividad de lectura demasiado egoísta y superficial; ... yo leía para mí, y lo que encontraba relacionado con lo que yo pensaba, era, y ahí se quedaba el texto; y si me gustaba, bien, hasta ahí llegaba el texto; y si no me gustaba, no leía más. En este momento creo que tengo una posición menos egoísta, porque me intereso más por el autor; por saber qué me está expresando, qué me está queriendo decir; por ampliar mi mundo; por no desechar un texto porque no me gustó, sino encontrar qué hay en ese texto... Entonces creo que he generado como una cierta solidaridad con el autor en la actividad de lectura; que me interesa entenderlo... es una cuestión de comunicarse (Tutor 2, entrevista en profundidad, noviembre de 2017). 
Es tanta la potencia que tiene el curso, para desarrollar... la reflexión, el análisis discursivo, el lenguaje como tal..., sí el desarrollo de la lengua, habilidades para escribir, para calificar, para también reflexionar qué es lo que quiere decir el estudiante; en cambio cuando tú estás con otras formas [de enseñar]... que el libro... haz estas hojitas, este vocabulario, la lista, pues son cosa que el docente ya sabe, y que no te permiten desarrollar otras habilidades. (Tutor 3, entrevista en profundidad, noviembre de 2017).

Los datos de las conversaciones con estos tutores son muy positivos, y siempre ha sido así en nuestros estudios; igual pasa con los estudiantes. Sin embargo, hemos mantenido discusiones permanentes en el desarrollo de las diferentes versiones del curso, y de ellas se destacan temas recurrentes acerca de posibles debilidades del curso y formas de mejorarlo. Desde el comienzo, por ejemplo, lo más difícil ha sido trabajar con estudiantes que no saben nada de inglés. El curso los enfrenta a materiales auténticos desde el primer día, y siempre hay un pequeño grupo que rechaza este reto y se retira sin darse la oportunidad de arrancar. Son también siempre muchos más los que se motivan por ver algo nuevo en su normalmente larga y generalmente fallida experiencia con el aprendizaje del inglés, los que tienen la paciencia y el deseo suficientes y soportan el esfuerzo que deben hacer al comenzar. Esto los ayuda a desarrollar la confianza que necesitan en sus tutores y en sus propias habilidades para poder partir de lo auténtico, y siempre lo logran, lo cual dice mucho de la calidad de los tutores y sus habilidades para promover esa confianza.

Igualmente se discute siempre que el trabajo gramatical podría sistematizarse más, a pesar de que se reconoce que el material auténtico nunca va a poder organizarse para practicar gramática específica. Siempre se decide seguir desarrollando la habilidad necesaria para encontrar en el material real aquellos aspectos del sistema de la lengua que vale la pena enfatizar y repasar cada vez que aparecen; los tutores deben seguir desarrollando habilidades para dar apoyo y pequeñas explicaciones a los estudiantes, de modo que vayan entendiendo paulatinamente cómo funciona la lengua y aumentando su repertorio del inglés.

La debilidad más grande que identifican algunos tutores en el curso es en el desarrollo de la habilidad oral, la más difícil de trabajar en contextos en los que la lengua no se usa en la vida diaria. En todas las estaciones se habla con los estudiantes del material, y la idea es que puedan usar el lenguaje incluido en él en sus respuestas, sus comentarios y preguntas propias. Pero para una minoría de los tutores falta más trabajo de habla espontánea, que consideran importante a partir de sus propias experiencias de aprendizaje del inglés. 
Es probable que aparezcan estas y otras debilidades cada vez que se dicte el curso, dado que cada versión es una nueva creación que utiliza los principios del aprendizaje y de la comunicación en que se basa, para el diseño de una experiencia que debe actualizarse con nuevo material de internet y adaptarse a cada nuevo grupo de estudiantes y sus necesidades. Además, en sus diferentes versiones el curso solo se ha dictado a niveles principiante e intermedio bajo, de modo que falta mucha experiencia por ocurrir. Quienes usamos esta metodología enfrentamos situaciones nuevas cada vez y estamos en permanente aprendizaje, aspecto que es posiblemente el más positivo de la experiencia, siempre que sucede.

\section{Conclusión}

No es pretensión de esta reflexión presentar un curso de inglés (con versiones para el desarrollo de habilidades de lenguaje en español) como la panacea de la enseñanza-aprendizaje de lenguas extranjeras en nuestro país. Es apenas una innovación posible en esta área, importante y necesitada de cambio actualmente aquí. Se ha presentado como ejemplo de que la formación en un marco teórico que reúne la teoría constructivista del aprendizaje humano y la del lenguaje como instrumento de comunicación y la práctica de la enseñanza-aprendizaje de lenguas dentro de una innovación pedagógica enseña a nuestros futuros profesores tanto lengua como pedagogía innovadora.

Definitivamente seguimos abogando por la enseñanza-aprendizaje de lengua extranjera ( $y$ aun del nativo español) por medio de desempeños comunicativos auténticos. Esto incluye el uso de materiales auténticos y la eliminación de tantos textos comerciales que les impiden a nuestros formadores de maestros y maestros en formación pensar y crear innovaciones efectivas para nuestros contextos. Pensamos y hemos logrado producir evidencia de que hacerlo así, y además poner en conexión las dos lenguas de nuestros aprendices de lengua y de pedagogía, puede formar verdaderos bilingües en nuestro contexto monolingüe.

Y más allá de las lenguas mismas, nuestros maestros necesitan bases para crear formas de ayudar a otros a aprender lenguas, que puedan adaptarse realmente a la diversidad de estudiantes que pueden tener en contextos como el nuestro. 


\section{Referencias}

200 extranjeros comienzan a dar clases de inglés en colegios públicos (2015, 2 de julio). El Tiempo. Recuperado de http://www.eltiempo. $\mathrm{com} /$

Ausubel, D. P. (1968). Educational psychology: A cognitive view. Nueva York: Holt, Rinehart and Winston.

Boix Mansilla, V. y Gardner, H. (1998). What are the qualities of understanding? En M. S. Wiske (ed.), Teaching for understanding (pp. 161196). San Francisco: Jossey-Bass.

Bonilla, S. y Cruz-Arcila, F. (2013). Sociocultural factors involved in the teaching of English as foreign language in rural areas of Colombia: An analysis of the impact on teachers' professional development. Research in Teacher Education, 3(2), 28-33.

Braine, G. (2013). Non-native educators in English language teaching. Londres: Routledge.

Bruffee, K. (1999). Collaborative learning, higher education, interdependence, and the authority of knowledge. Baltimore: The Johns Hopkins University Press.

Brumfit, C. J. y Johnson, K. (1991). The communicative approach to language teaching. Hong Kong: Oxford University Press.

Carretero, M. (2001). Constructivismo y educación. Buenos Aires: Grupo Editorial Aique.

Castilla, B. y Lobo-Guerrero, C. H. (1979). Marco teórico de los materiales Reading and Thinking in English (documento inédito). Bogotá: Universidad de los Andes.

Centro Virtual de Noticias de la Educación (2015). 200 nuevos nativos llegan al país para integrar equipo de Colombia Bilingüe. Recuperado de https://www.mineducacion.gov.co/cvn/1665/w3-article-351513. $\mathrm{html}$

Coll, C., Martín, E., Mauri, T., Miras, M., Onrubia, J., Solé, I. y Zabala, A. (1993). El constructivismo en el aula. Barcelona: Graó.

De Mejía, A. M., Ordóñez, C. L. y Fonseca, L. (2006). Estudio investigativo sobre el estado actual de la educación bilingüe (inglés-español) en Colombia. Recuperado de http://www.colombiaaprende.edu.co/ html/productos/1685/articles-266111_archivo_1.pdf

De Mejía, A.-M. y Fonseca, L. (2008). Orientaciones para políticas bilingües y multilingües en lenguas extranjeras en Colombia. Recuperado de http://www.colombiaaprende.edu.co/html/home/1592/articles-251339_pdf.pdf

Dewey, J. (1945). Experiencia y educación (trad. L. Luzuriaga). Buenos Aires: Losada. 
Dewey, J. (1991). How we think. Amherst, Nueva York: Prometheus Books.

Dickelman, G., \& Greenberg, J. D. (2000). Distributed cognition: A foundation for performance support. Performance Improvement, 39(6), 18-24.

Fichtner, F. y Chapman, K. (2011). The cultural identities of foreign language teachers. L2 Journal, 3(1), 116-140. Recuperado de http://repositories.cdlib.org/uccllt/l2/vol3/iss1/art6/

Gardner, H. (1991). The unschooled mind: How children think and how schools should teach. Nueva York: Basic Books.

Gómez Sará, M. (2017). Review and analysis of the Colombian foreign language bilingualism policies and plans. How Journal, 24(1), 139156. DOI:http://dx.doi.org/10.19183/how.24.1.343

González, Y. (2008). Relación entre las habilidades en lengua materna y el aprendizaje del inglés en niños de primero de primaria (tesis de maestría). Universidad de los Andes, Bogotá, Colombia.

González, A. (2010). English and English teaching in Colombia. En Kirkpatrick (ed.), The Routledge Handbook of World Englishes (pp. 332351). Londres: Routledge.

Guevara, D. C. y Ordóñez, C. L. (2012). Teaching English to very young learners through authentic communicative performances. Colombian Applied Linguistics Journal, 14(2): 9-27.

Hetland, L., Hammerness, K., Unger, C. y Wilson, D. (1998). How do students demonstrate understanding? En M. S. Wiske (ed.), Teaching for understanding (pp. 197-232). San Francisco: Jossey-Bass.

Insuasty, E. A. (2008, October). The place of authentic materials in language teaching: A historical perspective. Asocopi Newsletter, 8-9.

Jiménez, M. L. (2006). Una experiencia pedagógica para iniciar la formación del concepto de audiencia en niños escritores inexpertos (tesis de maestría). Universidad de los Andes, Bogotá, Colombia.

Kirkpatrick, A. (2014). World Englishes. En C. Leung y B. Street (eds.). The Routledge Companion to English Studies (pp. 33-45). Londres: Routledge.

Lastra, S. P. (2009). An understanding of the concept and conditions of bilingualism: A study in an EFL setting. HOW Journal, 16(1), 167-187.

Llano, A. M. (2009). Aprendizaje reflexivo para la autonomía en el aprendizaje de lengua extranjera en futuros profesores de inglés (tesis de maestría). Universidad de los Andes, Bogotá, Colombia.

Moussu, L. y Llurd, E. (2008). Non-native English-speaking English language teachers: History and research. Language Teaching, 41(3), 315-348. DOI:10.1017/S0261444808005028. 
Moya, M. R. (2008). La adquisición de competencia gramatical en lengua extranjera en aprendices adultos de inglés (tesis de maestría). Universidad de los Andes, Bogotá, Colombia.

Ninio, A, y Snow, C. E. (1996). Pragmatic Development. Boulder, Colorado: Westview Press (Harper Collins).

Nunan, D. (1989). Designing tasks for the communicative classroom. Cambridge, UK: Cambridge University Press.

Ordóñez, C. L. (2000). Oral bilingual proficiency of Colombian adolescents(tesis doctoral inédita). Harvard Graduate School of Education, Cambridge, MA, EE. UU.

Ordóñez, C. L. (2004a). Pensar pedagógicamente desde el constructivismo. De las concepciones a las prácticas pedagógicas. Revista de Estudios Sociales, 19, 7-12.

Ordóñez, C. L. (2004b). EFL and native Spanish in elite bilingual schools in Colombia: A first look at bilingual adolescent frog stories. International Journal of Bilingual Education and Bilingualism, Special Issue: Bilingual Education in South America, 7(5), 449-474.

Ordóñez, C. L. (2005). Oral bilingual proficiency of Colombian adolescents in an elite bilingual school. En J. Cohen, K. McAllister, K. Rolstad y J. MacSwan (eds.). ISB4: Proceedings of the Fourth International Symposium on Bilingualism (pp. 1765-1783). Somerville, MA: Cascadilla Press.

Ordóñez, C. L. (2006). Pensar pedagógicamente, de nuevo, desde el constructivismo. Revista Ciencias de la Salud, 4 [Especial], 14-23.

Ordóñez, C. L. (2008a). Currículo conectado de español e inglés para el Colegio Bilingüe San Bonifacio de las Lanzas, de Ibagué. Documento inédito.

Ordóñez, C. L. (2008b). Education for bilingualism in international languages in a monolingual socio-linguistic context. Lenguaje, 36(2), 253-384.

Ordóñez, C. L. (2010a). Desempeño auténtico en educación. En C. L. Ordóñez y C. Castaño, Pedagogía y didáctica: libro del maestro (p. 151-160). Quito: Ministerio de Educación de Ecuador.

Ordóñez, C. L. (2010b). Educación para el bilingüismo en contexto monolingüe: dos lenguas conectadas desde el currículo. Elia: Estudios de Lingüística Inglesa Aplicada, 10, 41-76.

Ordóñez, C. L. (2011). Education for bilingualism: Connecting Spanish and English from the curriculum, into the classroom, and beyond. Profile 13(2), 147-161.

Ordóñez, C. L. (2012). Educación para el bilingüismo y aprendizaje de maestros: comprensión del desempeño auténtico en la acción de cambiar prácticas pedagógicas. Folios, 36, 3-22. 
Ordóñez, C. L. (2015a). Bilingüismo en contexto monolingüe: contra la corriente. Miríada Hispánica 10, 107-126.

Ordóñez, C. L. (2015b). Innovación e investigación en la formación de profesores de inglés en la Universidad Nacional de Colombia. En L. Ochoa (ed.). Investigación e innovación educativas: docentes (pp. 39-65). Bogotá: Universidad Nacional de Colombia-Centro Editorial de la Facultad de Ciencias Humanas.

Ordóñez, C. L. (2016). Just singing, role playing and reading: A case study in education for bilingualism. En V. A. Murphy y M. Evangelou (eds.), Early childhood education in English for Speakers of Other Languages (pp. 233-240). Londres: British Council.

Ordóñez, C. L., Barriga-Villanueva, R., Snow, C. E., Uccelli, P., Shiro, M. y Schnell, B. (2001). Sintaxis y discurso: dos áreas de investigación en la adquisición del español oral. Revista Latina de Pensamiento y Lenguaje, 9(2), 131-163.

Perkins, D. (1998). What is understanding? En M. S. Wiske (ed.), Teaching for understanding (pp. 39-57). San Francisco: Jossey-Bass.

Perrone, V. (1998). Why do we need a pedagogy of understanding? En M. S. Wiske (ed.), Teaching for understanding (pp. 1338). San Francisco: Jossey-Bass.

Piaget, J. (1970). Piaget's theory. En P. H. Mussen (ed.), Carmichael's manual of child psychology (3 ${ }^{\text {rd }}$ ed., vol. 1, pp. 703-723). Nueva York: Wiley.

Rogoff, B. (1996). Apprenticeship in thinking. Cognitive development in social context. Nueva York: Oxford University Press.

Savery, J. y Duffy, T. (1996). Problem based learning: An instructional model and its constructivist framework. En B. Wilson (ed.), Constructivist learning environments: Case studies in instructional design (pp. 134-147). Englewood Cliffs, New Jersey: Educational Technology Publications.

Seidlhofer, B. (2004). Research perspectives on teaching English as a lingua franca. Annual Review of Applied Linguistics, 24, 209-239. DOI: 10.1017/S0267190504000145

Sierra, L. (2017). Propuesta curricular basada en desempeños auténticos comunicativos para el desarrollo de habilidades comunicativas académicas en español en estudiantes universitarios (tesis de maestría). Bogotá, Universidad Nacional de Colombia.

Tolosa, C., Ordóñez C. L. y Alfonso, T. (2015). Online peer feedback between Colombian and New Zealand FL beginners: A comparison and lessons learned. Profile: Issues in Teachers' Professional Development, 17(1), 73-86. 
Tolosa, C., Ordóñez, C. L. y Guevara, D. C. (2017). Language learning shifts and attitudes towards language learning in an on-line tandem program for beginner writers. Profile: Issues in Teachers' Professional Development, 19(1), 105-118.

Torres, I. C. (2006). Una iniciación a la escritura argumentativa: efectos de una intervención para enseñar a escribir un ensayo argumentativo a estudiantes de $10^{\circ}$ grado de un centro educativo distrital de Bogotá (tesis de maestría). Universidad de los Andes, Bogotá.

Varela, M. P. (2008). Adquisición escolar de español académico en niños de 7 años que construyen en colaboración torres y puentes de Lego (tesis de maestría). Universidad de los Andes, Bogotá.

Vygotsky, L. (1978). Mind in society. Cambridge, MA: Harvard University Press.

Widdowson, H. G. (1978). Teaching language as communication. Oxford: Oxford University Press. 